\title{
High-Performance Storage System for the LHCb Experiment
}

Sai Suman Cherukuwada, CERN Niko Neufeld, CERN

IEEE NPSS RealTime 2007, FNAL 
IHCD Background

- Large Hadron Collider beauty

- One of 4 Major CERN experiments at the LHC

- Single-arm Forward Spectrometer

o b-Physics, CP Violation in the Interactions of b-hadrons 


\section{LHCb Data Acquisition}

- Level 0 Trigger - 1 $\mathrm{MHz}$

- High Level Trigger

$-2-5 \mathrm{kHz}$

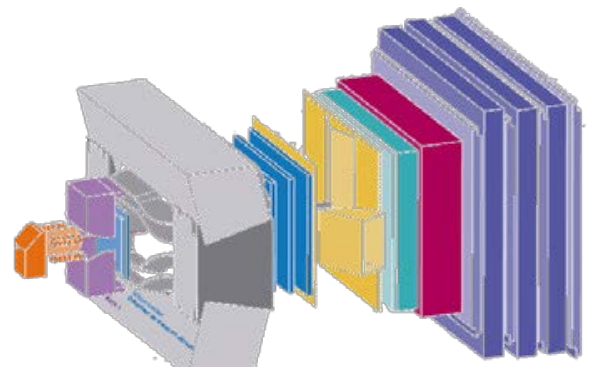

L0 Electronics
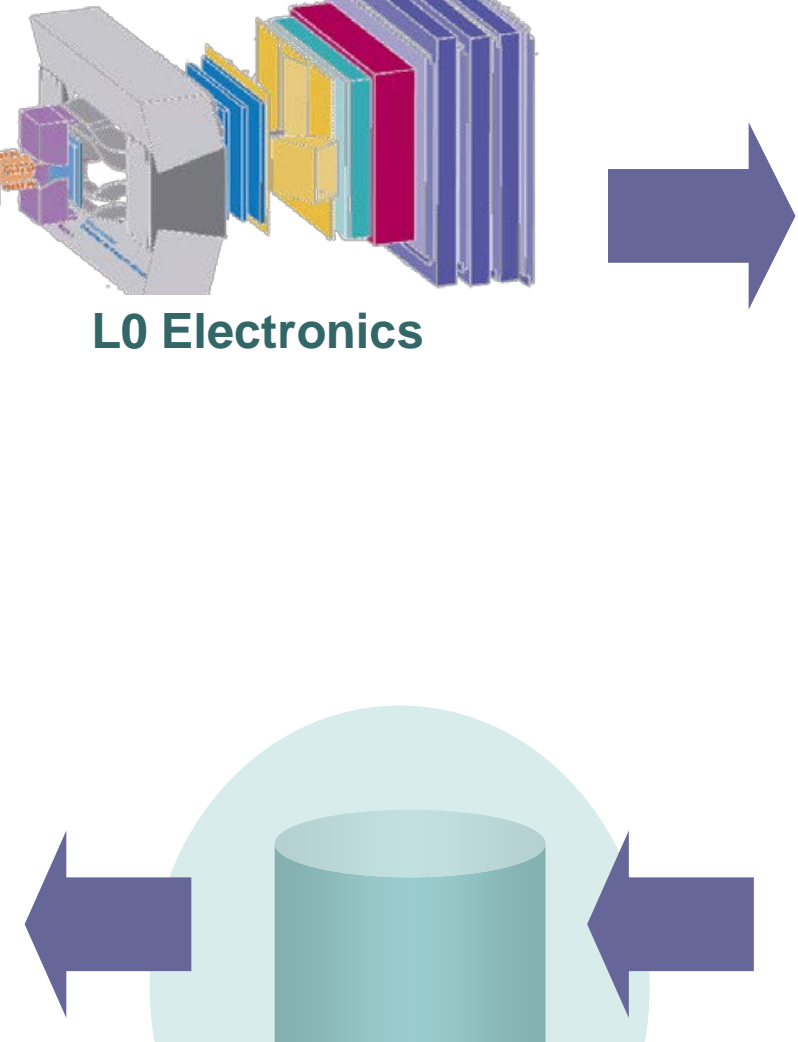

Storage at

Experiment Site
Readout Boards
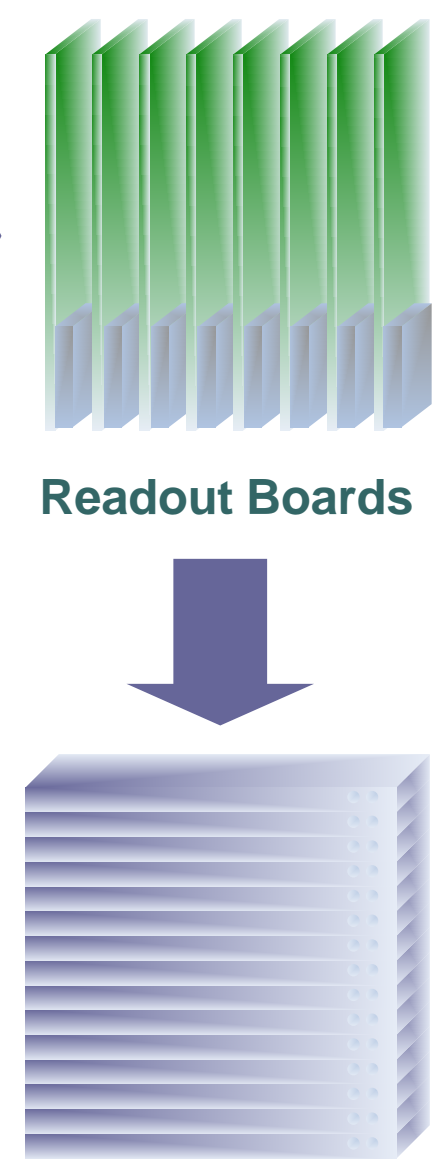

High-Level Trigger Farm 


\section{Storage Requirements}

- Must sustain Write operations for 2-5 kHz of Event Data at $\sim 30 \mathrm{kB} / \mathrm{event}$, with peak loads going up to twice as much

- Must sustain matching read operations for staging out data to tape centre

- Must support reading of data for Analysis tasks

- Must be Fault tolerant

- Must easily scale to support higher storage capacities and/or throughput 


\section{Architecture : Choices}

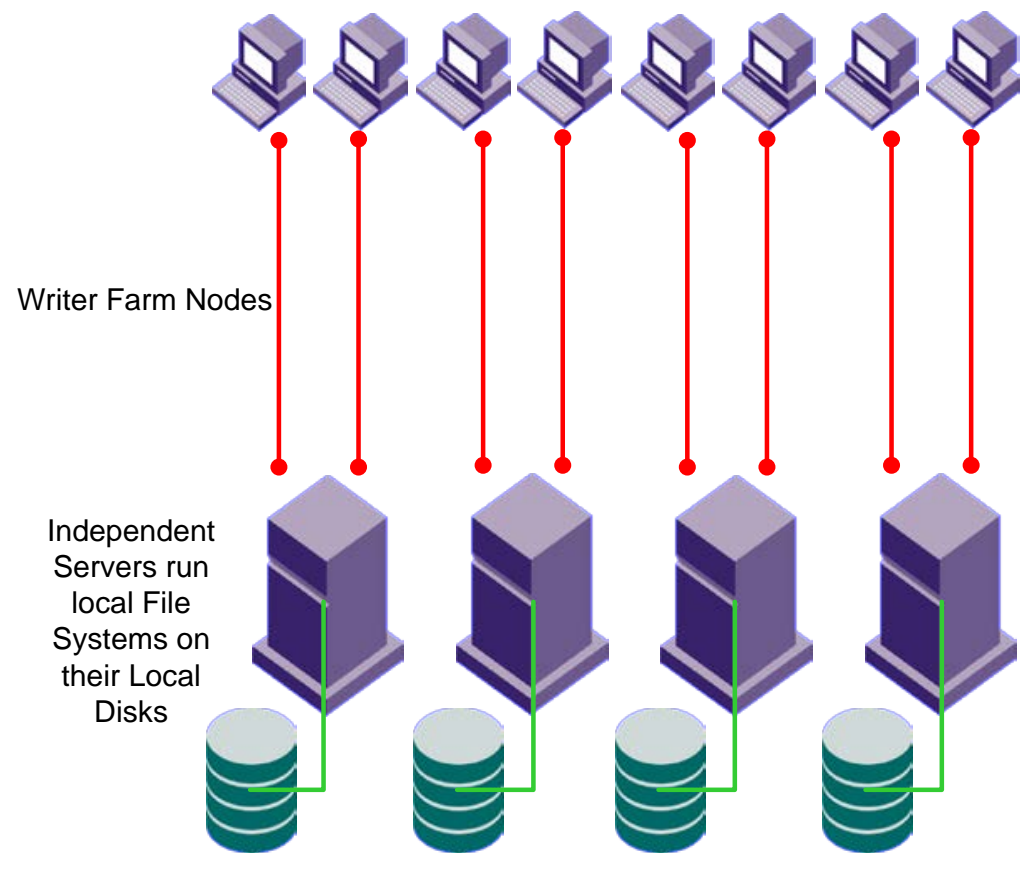

Fully Partitioned Independent Servers

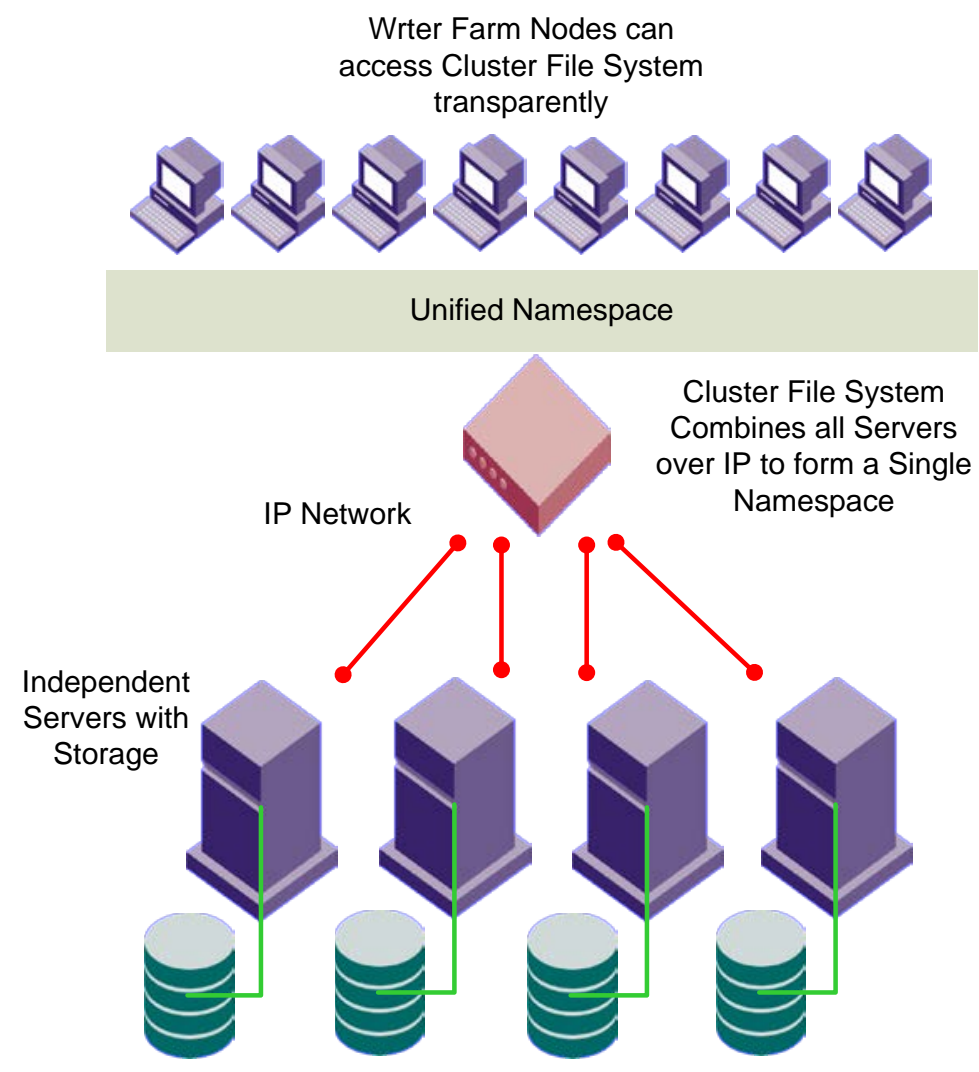

Cluster File System 


\section{Architecture : Shared-Disk File System Cluster}

- Can Scale Compute or Storage Components independently

- Locking Overhead restricted to very few servers

- Unified namespace is simpler to manage

- Storage fabric delivers high throughput

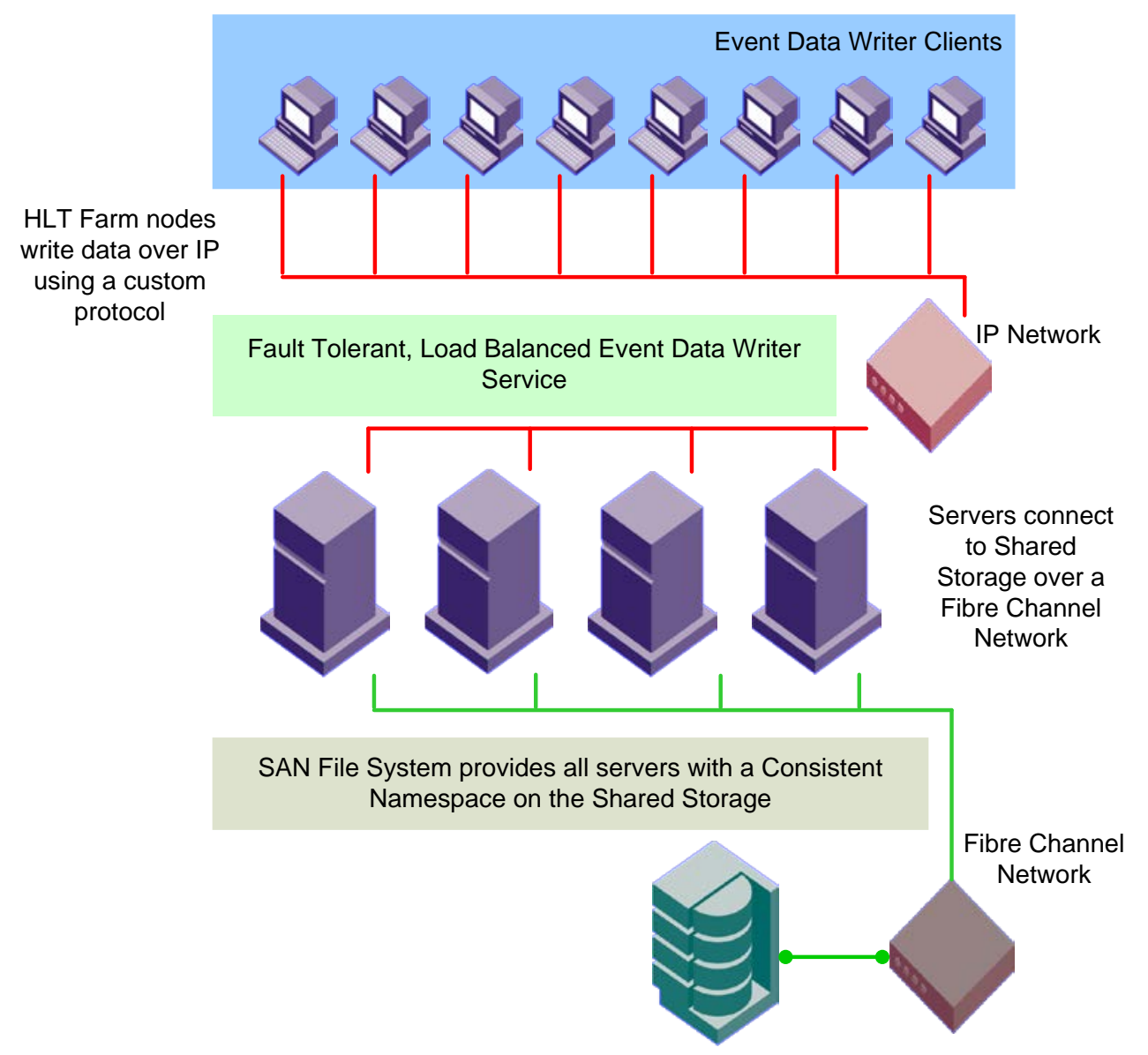

Shared-Disk File System Cluster 


\section{Hardware : Components}

- Dell PowerEdge 2950 Intel Xeon Quad Core Servers $(1.6 \mathrm{GHz})$ with $4 \mathrm{~GB} F B D$ RAM

- QLogic QLE2462 4 Gbps Fibre Channel Adapters

- DataDirect Networks S2A 8500 Storage Controllers with 2 Gbps host-side ports

- 50 x Hitachi 500 GB 7200 rpm SATA Disks

- Brocade 200E 4 Gbps Fibre Channel Switch 


\section{Hardware : Storage}

\section{Controller}

- DirectRAID

- Combined Features of RAID3, RAID5, and RAID0

- $8+1 p+1 s$

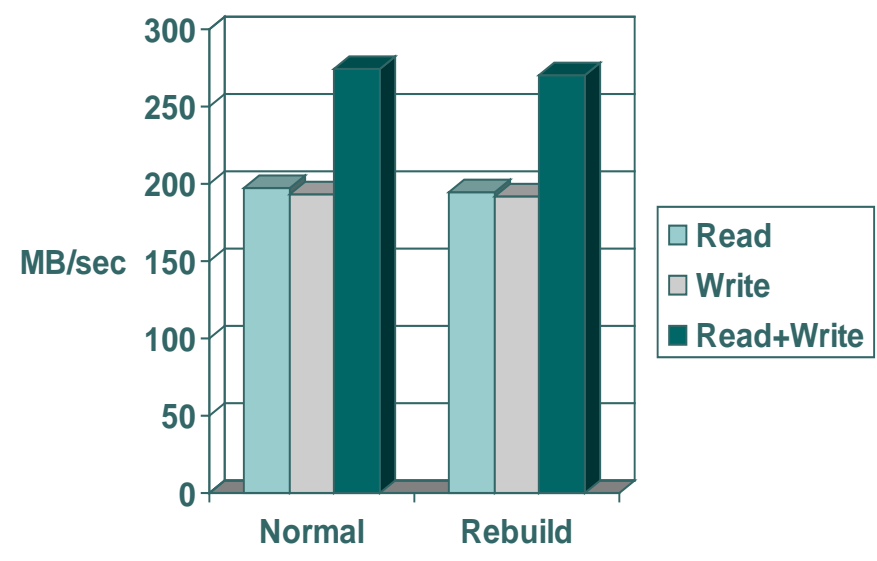

- Very Low Impact on Disk Rebuild

- Large Sector Sizes (up to $8 \mathrm{kB}$ ) supported

- Eliminates host-side striping

-IOZone File System Benchmark with 8 threads writing 2 GB files each on one server

-Tested first in "Normal" mode with all disks in normal health, and then in "Rebuild", with one disk in the process of being replaced by a global hot spare 


\section{Software}

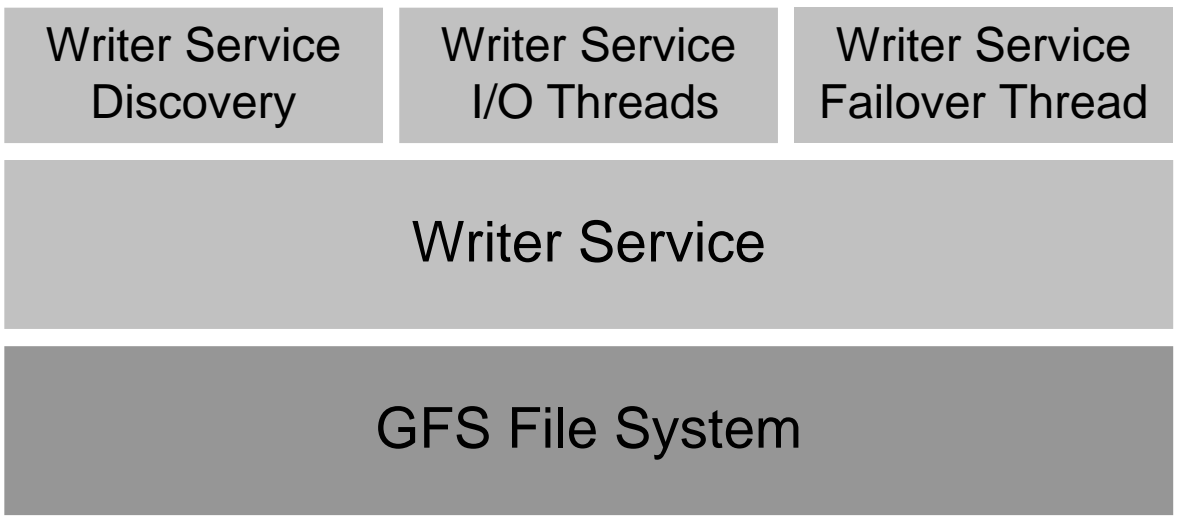

\section{Linux Logical Volume Manager}

Linux Multipath Driver

$\Longleftarrow \Longleftarrow \begin{gathered}\text { SCSI LUNs } \\ \text { (Logical Units) }\end{gathered}$




\section{Software : Shared-Disk File}

\section{System}

- Runs on RAID volumes exported from Storage Arrays (called LUNs or Logical Units)

- Can be mounted by multiple servers simultaneously

- Lock Manager ensures consistency of operations

- Scales almost linearly up to 4 nodes (at least)

-IOZone Test with 8 threads, O_DIRECT I/O -LUNs striped over 100+ disks

-2 Gbps Fibre Channel Connections to Disk Array
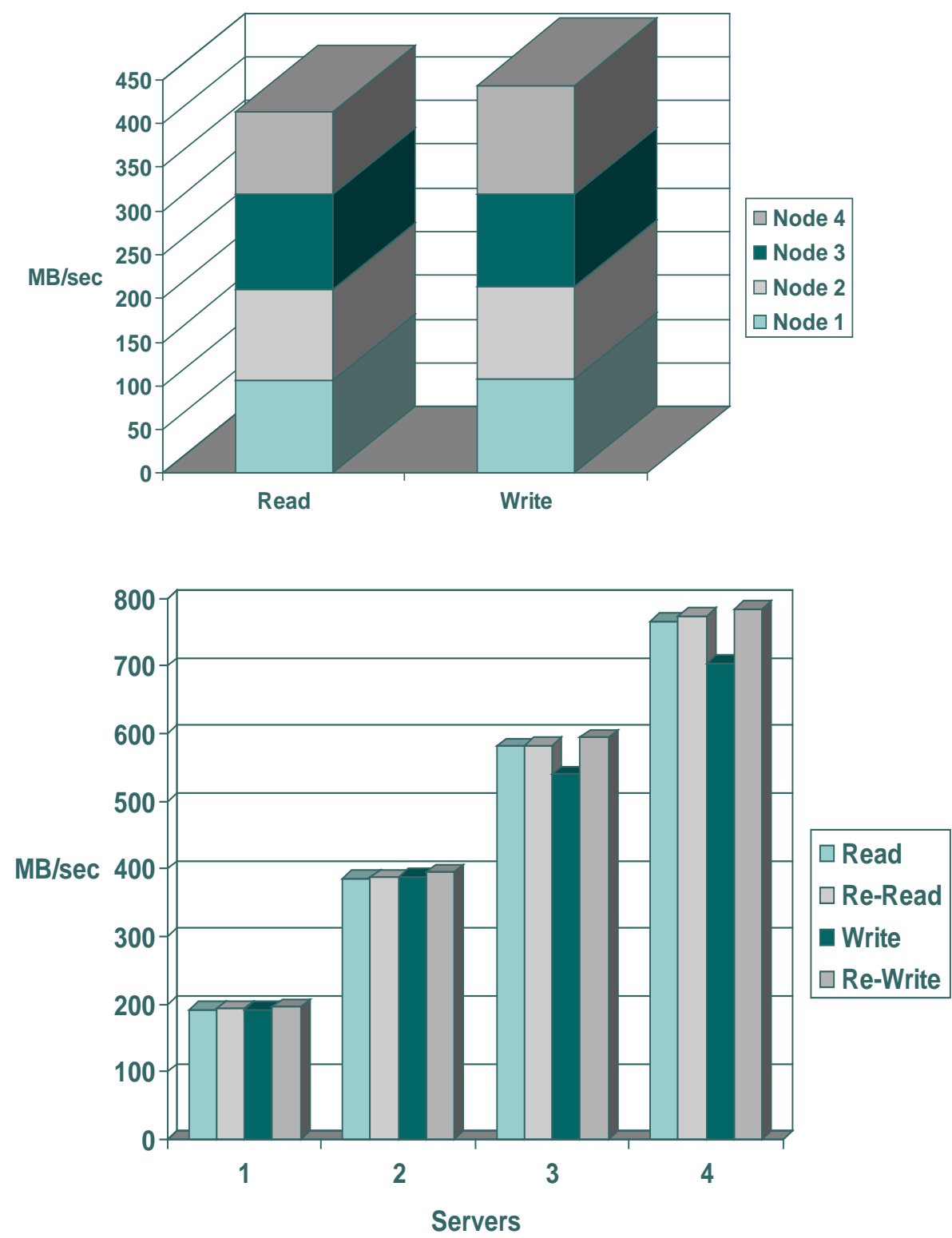

Sai Suman Cherukuwada and Niko Neufeld, CERN 


\section{Writer Service : Design}

Goals

- Enable a large number of HLT Farm Servers to write to disk

- Write Data to shared disk file system at close to maximum disk throughput

o Failover + Failback with no data loss

- Load Balancing between instances

- Write hundreds of concurrent files per server 


\section{Writer Service : Discovery}

- Discovery and status updates performed through multicast

\section{Writer Service 1}

- Service Table maintains current status of all known hosts

- Service Table contents constantly updated to all connected Gaudi Writer Processes from the HLT Farm

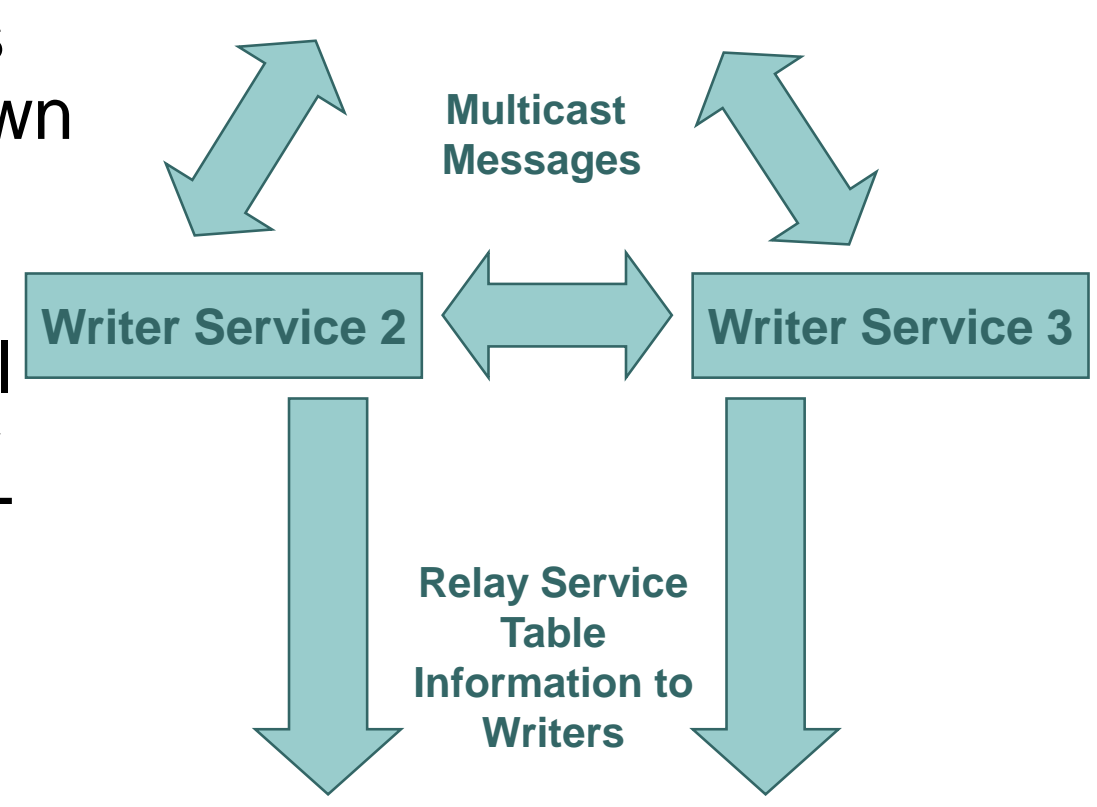




\section{Writer Process : Writing}

- Cache every event

o Send to Writer Service

- Wait for Acknowledgement

o Flush and free 


\section{Writer Service : Failover}

- Writer Processes aware of all instances of Writer Service

- Each Data Chunk is entirely self-contained

- Writing of a Data Chunk is idempotent

- If Writer Service fails, Writer Process can reconnect and resend

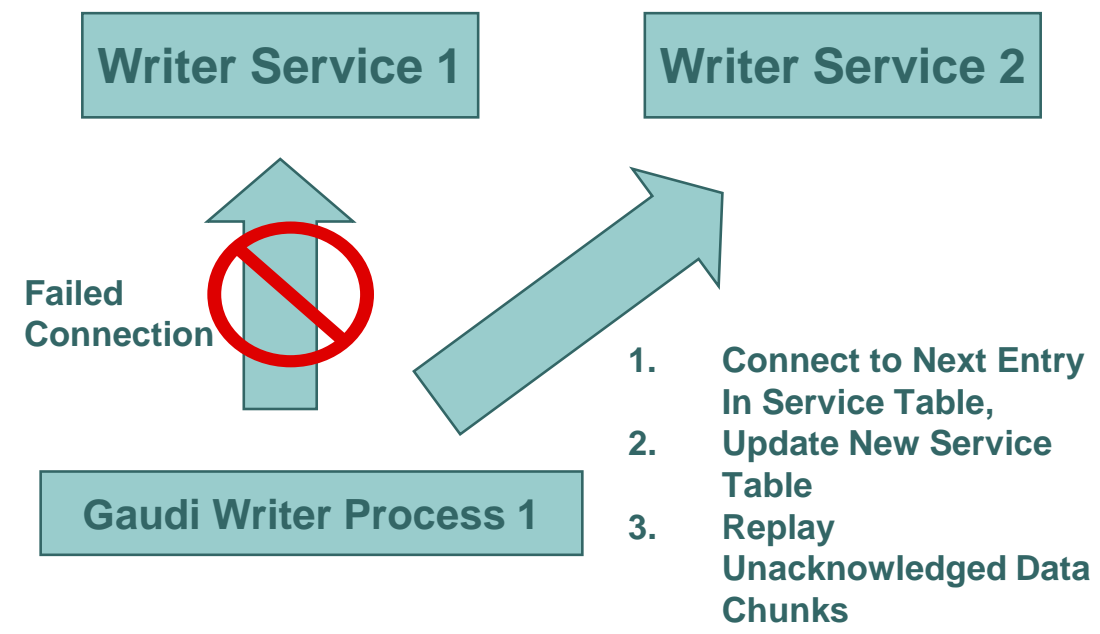
unacknowledged chunks 


\section{Writer Service : Throughput}

- Cached concurrent write performance for large numbers of files is insufficient

- Large CPU and memory load (memory copy)

- O_DIRECT reduces CPU and memory usage

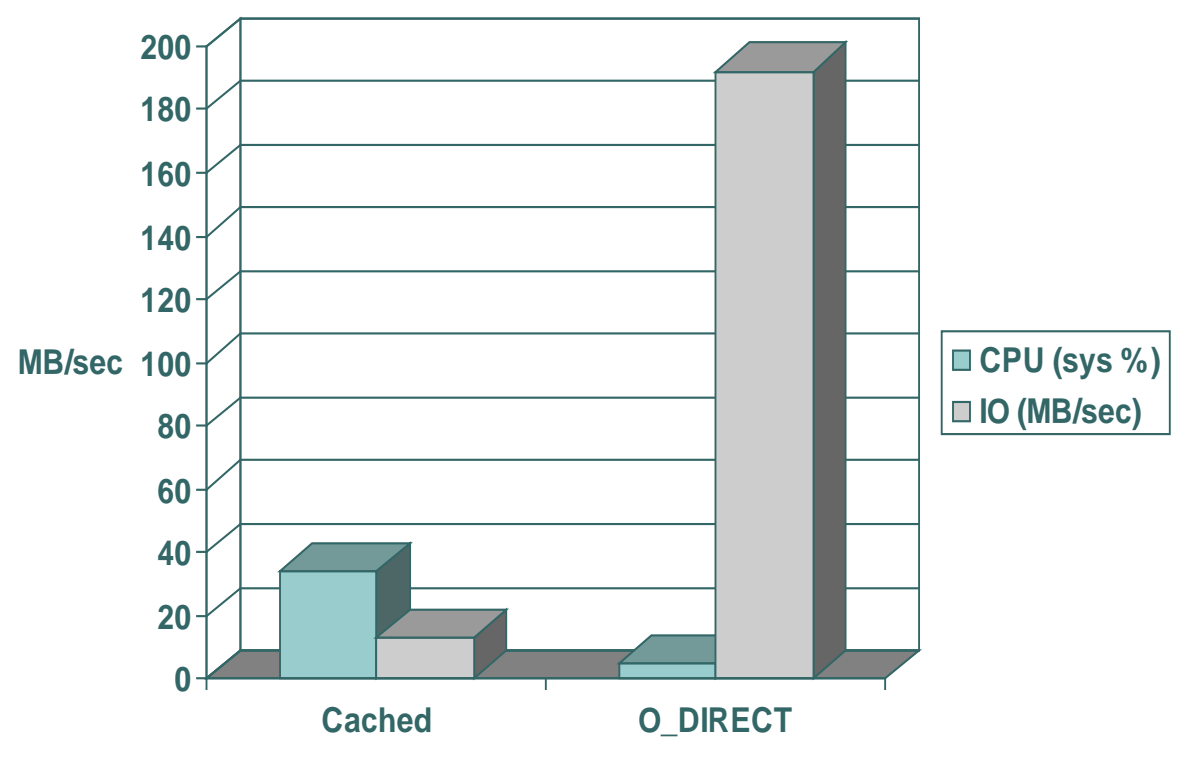

- Data need to be pagealigned for O_DIRECT

- Written event data are not aligned to anything

-Custom test writing 32 files per thread $\times 8$ threads -Write sizes varying from 32 bytes to $1 \mathrm{MB}$ -LUNs striped over 16 disks

-2 Gbps Fibre Channel Connections to Disk Array 


\section{Writer Service : Throughput per Server}

- Scales up with number of clients

- Write throughput within $3 \%$ of maximum achievable through GFS

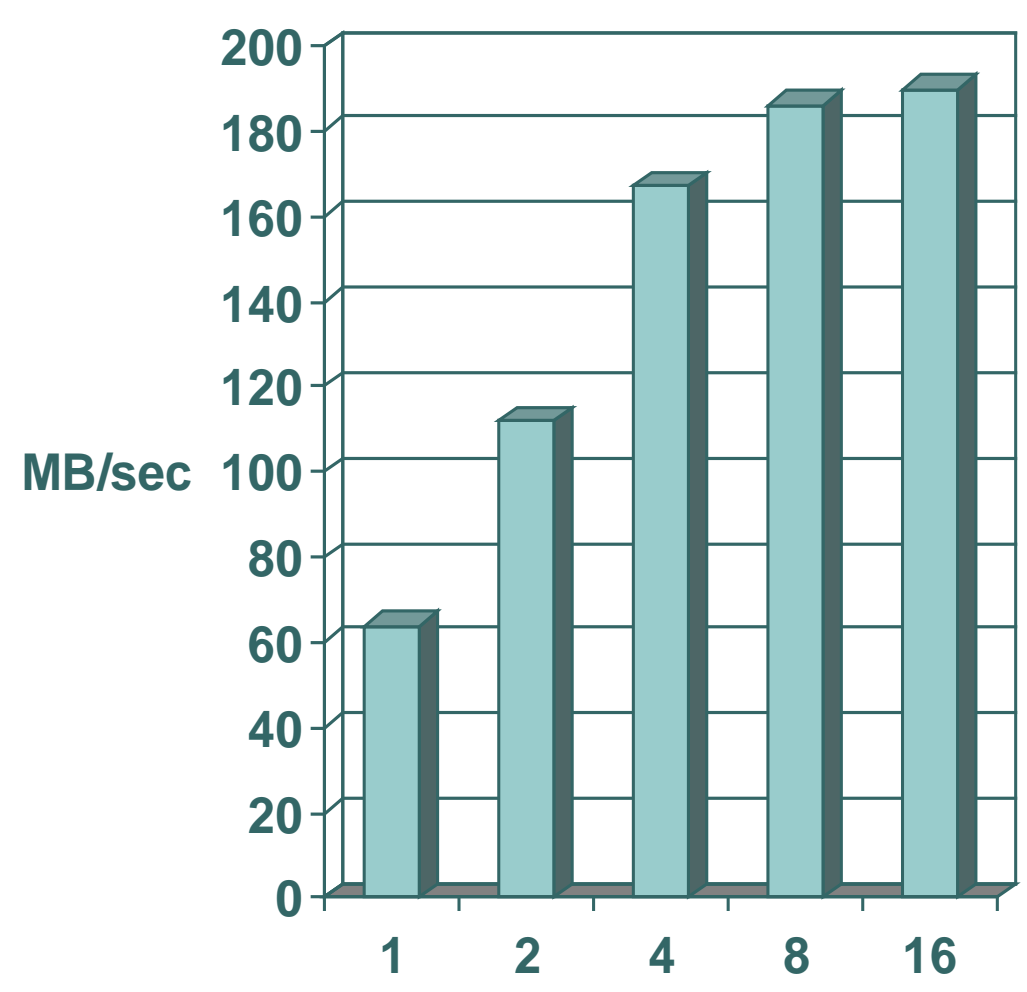

-Custom test writing event sizes ranging from 1 byte to $2 \mathrm{MB}$

Clients

-LUNs striped over 16 disks

-2 Gbps Fibre Channel Connections to Disk Array

-2 11 Gbps Ethernet Connections to Server

-CPU Utilisation 7-10\% 


\section{Conclusions \& Future Work}

- Solution that can offer high read and write throughput with minimal overhead

- Can be scaled up easily with more hardware

o Failover with no performance hit

- More sophisticated "Trickle" load-balancing algorithm in the process of being prototyped

- Maybe worth implementing a full-POSIX FS version someday? 

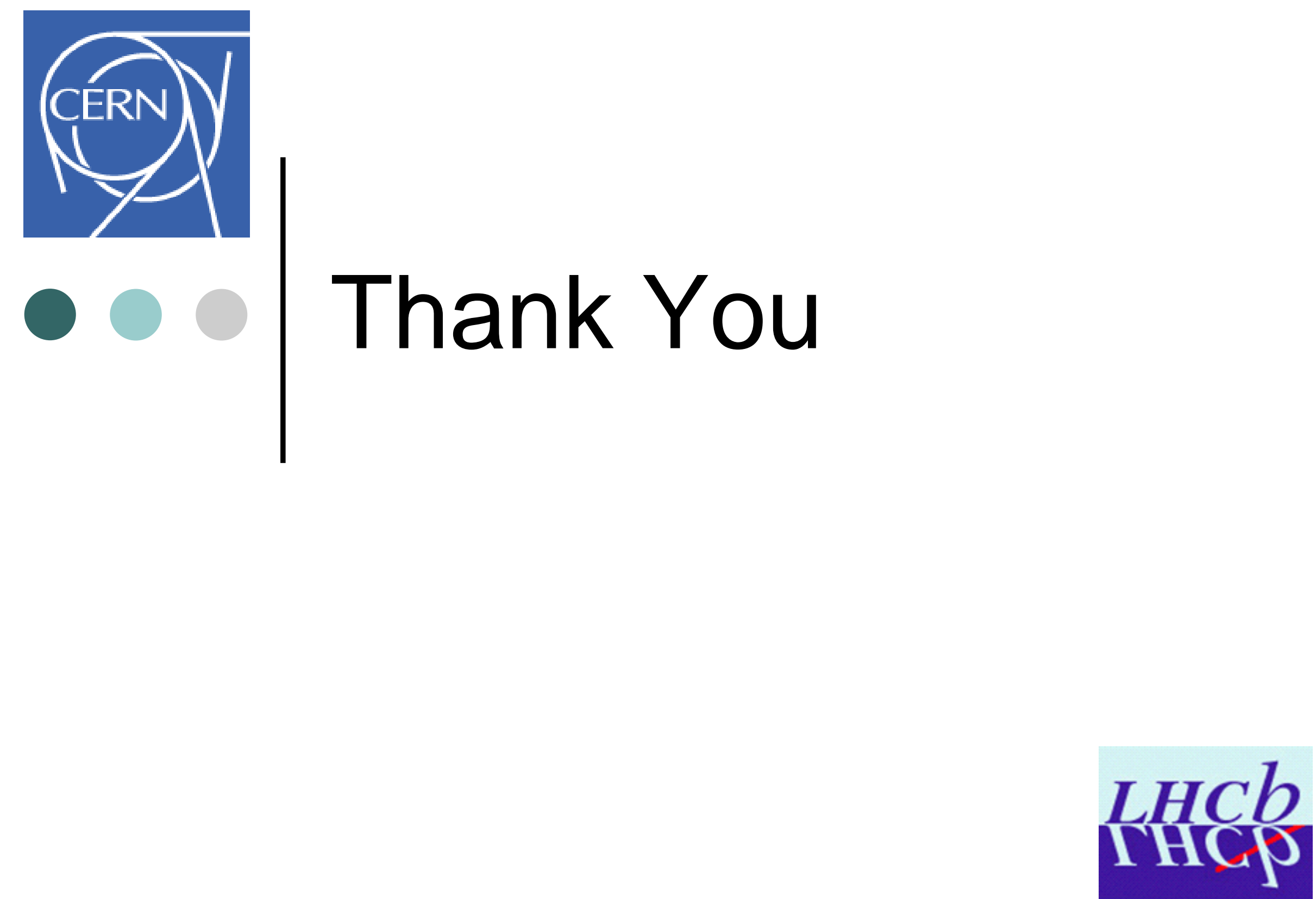

DNLINE 


\section{Writer Service}

- Linux-HA not suited for Load Balancing

- Linux Virtual Server not suited for Write Workloads

- NFS in sync mode too slow, async mode can lead to information loss on failure

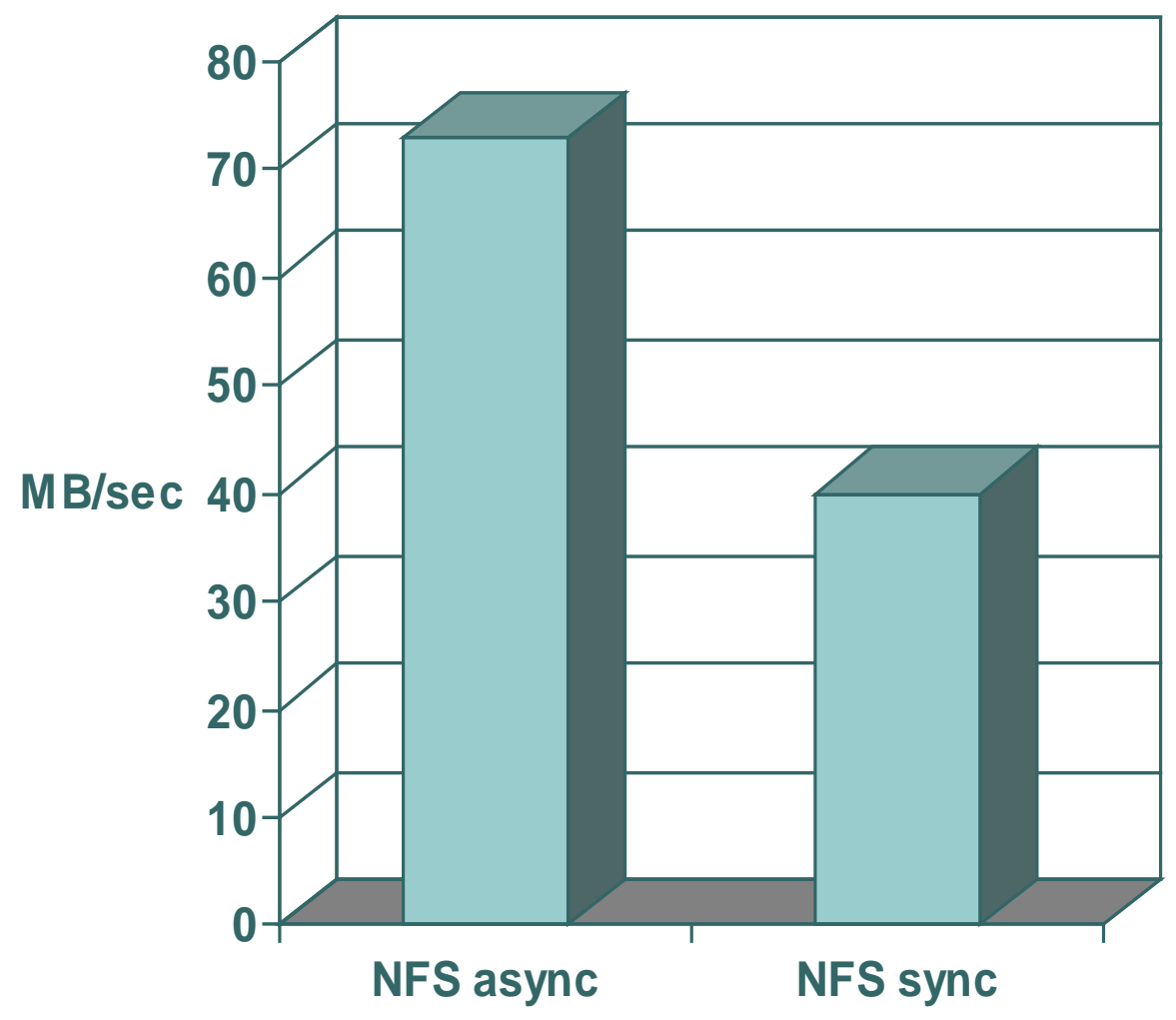

